\title{
Extending statistical learning for aneurysm rupture assessment to Finnish and Japanese populations using morphology, hemodynamics, and patient characteristics
}

\author{
Felicitas J. Detmer, MSc, ${ }^{1}$ Sara Hadad, MSc, ${ }^{1}$ Bong Jae Chung, PhD, ${ }^{2}$ Fernando Mut, PhD, ${ }^{1}$ \\ Martin Slawski, PhD, ${ }^{3}$ Norman Juchler, MSc,,${ }^{4,5}$ Vartan Kurtcuoglu, PhD, ${ }^{5}$ Sven Hirsch, $\mathrm{PhD},{ }^{4}$ \\ Philippe Bijlenga, MD, ${ }^{6}$ Yuya Uchiyama, BSc, ${ }^{7,8}$ Soichiro Fujimura, MSc, ${ }^{7,8}$ \\ Makoto Yamamoto, PhD, ${ }^{9}$ Yuichi Murayama, MD, ${ }^{10}$ Hiroyuki Takao, MD, PhD, 7,8,10 \\ Timo Koivisto, MD, PhD, ${ }^{11}$ Juhana Frösen, MD, PhD, ${ }^{11}$ and Juan R. Cebral, PhD ${ }^{1}$
}

${ }^{1}$ Bioengineering Department and ${ }^{3}$ Statistics Department, George Mason University, Fairfax, Virginia; ${ }^{2 D e p a r t m e n t ~ o f ~}$ Mathematical Sciences, Montclair State University, Montclair, New Jersey; ${ }^{4}$ Institute of Applied Simulation, ZHAW University of Applied Sciences, Wädenswil, Switzerland; ${ }^{5}$ The Interface Group, Institute of Physiology, University of Zürich, Switzerland; ${ }^{6}$ Clinical Neurosciences Department, University of Geneva, Switzerland; ${ }^{7}$ Graduate School of Mechanical Engineering, Tokyo University of Science, Tokyo, Japan; Departments of ${ }^{8}$ nnovation for Medical Information Technology and ${ }^{10}$ Neurosurgery, The Jikei University of Medicine, Tokyo, Japan; 'Department of Mechanical Engineering, Tokyo University of Science, Tokyo, Japan; and ${ }^{11} \mathrm{Hemorhagic} \mathrm{Brain}$ Pathology Research Group, Department of Neurosurgery, Kuopio University Hospital, Kuopio, Finland

\begin{abstract}
OBJECTIVE Incidental aneurysms pose a challenge for physicians, who need to weigh the rupture risk against the risks associated with treatment and its complications. A statistical model could potentially support such treatment decisions. A recently developed aneurysm rupture probability model performed well in the US data used for model training and in data from two European cohorts for external validation. Because Japanese and Finnish patients are known to have a higher aneurysm rupture risk, the authors' goals in the present study were to evaluate this model using data from Japanese and Finnish patients and to compare it with new models trained with Finnish and Japanese data.
\end{abstract}

METHODS Patient and image data on 2129 aneurysms in 1472 patients were used. Of these aneurysm cases, 1631 had been collected mainly from US hospitals, 249 from European (other than Finnish) hospitals, 147 from Japanese hospitals, and 102 from Finnish hospitals. Computational fluid dynamics simulations and shape analyses were conducted to quantitatively characterize each aneurysm's shape and hemodynamics. Next, the previously developed model's discrimination was evaluated using the Finnish and Japanese data in terms of the area under the receiver operating characteristic curve (AUC). Models with and without interaction terms between patient population and aneurysm characteristics were trained and evaluated including data from all four cohorts obtained by repeatedly randomly splitting the data into training and test data.

RESULTS The US model's AUC was reduced to 0.70 and 0.72 , respectively, in the Finnish and Japanese data compared to 0.82 and 0.86 in the European and US data. When training the model with Japanese and Finnish data, the average AUC increased only slightly for the Finnish sample (to $0.76 \pm 0.16$ ) and Finnish and Japanese cases combined (from 0.74 to $0.75 \pm 0.14$ ) and decreased for the Japanese data (to $0.66 \pm 0.33$ ). In models including interaction terms, the AUC in the Finnish and Japanese data combined increased significantly to $0.83 \pm 0.10$.

CONCLUSIONS Developing an aneurysm rupture prediction model that applies to Japanese and Finnish aneurysms requires including data from these two cohorts for model training, as well as interaction terms between patient population and the other variables in the model. When including this information, the performance of such a model with Japanese

ABBREVIATIONS AUC = area under the receiver operating characteristic curve; $B L=$ bulge location; $C F D=$ computational fluid dynamics; $H W R=$ height/width ratio; $I A=$ intracranial aneurysm; KE = kinetic energy; LSA = low shear area; MLN = mean surface curvature; NSI = nonsphericity index; OSImax = maximum oscillatory shear stress; $\mathrm{SAH}=$ subarachnoid hemorrhage; WSS = wall shear stress.

SUBMITTED February 28, 2019. ACCEPTED April 9, 2019.

INCLUDE WHEN CITING DOI: 10.3171/2019.4.FOCUS19145. 
and Finnish data is close to its performance with US or European data. These results suggest that population-specific differences determine how hemodynamics and shape associate with rupture risk in intracranial aneurysms.

https://thejns.org/doi/abs/10.3171/2019.4.FOCUS19145

KEYWORDS cerebral aneurysm; rupture; risk; hemodynamics; morphology

I NTRACRANIAL aneurysms (IAs) are a common vascular pathology, affecting about $2 \%-3 \%$ of the population. ${ }^{18,20}$ Most IAs remain asymptomatic and do not cause any medical complications. In cases of aneurysm rupture, however, the patient suffers from subarachnoid hemorrhage (SAH), which is associated with high mortality and morbidity as well as a large economic burden..$^{19,21}$ Given the increased use of medical imaging for the diagnosis of various diseases, a rising number of IAs are incidentally diagnosed. In these cases, physicians face the challenge of having to decide whether to treat the patient or to conservatively follow up the IA since the risk associated with interventions aimed at preventing future aneurysm rupture outweighs the natural aneurysm rupture risk. ${ }^{12,17,23}$

The pathophysiological mechanisms leading to aneurysm rupture are not yet fully understood; however, a plethora of risk factors have been suggested in the literature. ${ }^{13}$ These risk factors include patient-related variables such as sex or smoking status, genetics, geometric factors describing the shape of an IA, and hemodynamic factors. Hemodynamics are believed to play an important role in aneurysm development, growth, and rupture through biomechanical signaling processes in the vessel wall.,16 Several risk scores for aneurysm growth or rupture have been published to support physicians in decision-making. ${ }^{2,10,11}$ These scores are mainly based on patient characteristics, and for a specific IA, only its location, size, and "irregularity," defined simply as an "irregular shape"2 or as an "irregularity or lobulation," 10 are taken into account. While classification of the aneurysm shape as simple or irregular is highly subjective, aneurysm hemodynamics are not considered at all. To overcome this limitation, we recently developed an aneurysm rupture probability model that takes into account hemodynamic, geometric, and patient-related factors. ${ }^{6}$ The model was developed using data on 1631 aneurysms and most of these cases (1614) were obtained from hospitals in the US. When evaluating the model using two external European cohorts, it showed good predictive performance, indicating that it generalizes to patient cohorts other than the one used for model training.?

The results of several studies have suggested that Finnish and Japanese populations have a higher risk of aneurysmal SAH than other populations. ${ }^{11,22}$ Comparison of aneurysm-related and patient-related characteristics between these two high-risk aneurysm populations and other aneurysm patient populations may provide insight into what causes the higher risk of aneurysmal SAH in the Finnish and Japanese. Such knowledge is likely to increase our overall understanding of the development and progression of the disease. Therefore, the aim of this study was to evaluate in Japanese and Finnish aneurysm patients the rupture probability model we originally developed utilizing US-derived data. Furthermore, that model was compared to a new model that was trained on data that included parts of the Finnish and Japanese data as well as the European (other than Finnish) and US data. Finally, the models' performances were compared to a similarity-based approach in which similar aneurysms from our database (US and European) were identified to classify a new Japanese or Finnish aneurysm's rupture status.?

\section{Methods \\ Patient and Image Data}

This study is based on four aneurysm data sets. For the remainder of this paper, they will be referred to as "US," "European" (i.e., European other than Finnish), "Finnish," and "Japanese." All four data sets included patient information (patient sex and age), as well as 3D images of the respective aneurysm and surrounding vasculature (3D rotational angiography, US, European, and Finnish cohorts; CTA, Japanese cohort). The US data were collected from hospitals mainly in the US and consist of 1631 aneurysms in 1061 patients. Details of the data set can be found in Detmer et al. ${ }^{6}$ The two European cohorts consist of a total of 249 aneurysms in 203 patients and were composed of the AneuX data set and the publicly available AneuRisk data set (see Detmer et al. ${ }^{7}$ for details). The Finnish data set consists of 102 IAs in 71 patients treated at Kuopio University Hospital. All the US, European, and Finnish data are cross-sectional data, for which the aneurysm rupture status was defined as the rupture status at the time of patient presentation at the hospital. In contrast, the Japanese data set is composed of data on 147 aneurysms in 137 patients, representing longitudinal data in which aneurysm rupture status was defined after a mean follow-up of 900.8 days in cases of ruptured aneurysms (all ruptures occurred during follow-up) and 2432.1 days in cases classified as unruptured aneurysms. The images used for hemodynamic and shape characterizations were obtained before and close to the rupture for ruptured cases. We used the most recent images for the unruptured cases.

Table 1 shows a comparison of patient and aneurysm characteristics among the four populations.

\section{Hemodynamic Modeling and Shape Characterization}

From the US and Finnish images, the aneurysms and surrounding vasculatures were segmented with in-house software based on a thresholding approach. The AneuX images had been segmented with a geodesic active regions method integrated in the@neuFuse software and a level set-based approach implemented in MATLAB (MathWorks), while the AneuRisk image segmentations had been generated using the Vascular Modeling Toolkit (VMTK) with a gradient-driven level set approach. ${ }^{7}$ For 
TABLE 1. Summary of patient characteristics and distribution of aneurysm location in the cerebral vasculature for the four data sets

\begin{tabular}{lcccc}
\hline \multicolumn{1}{c}{ Variable } & US* & European & Japanese & Finnish \\
\hline No. of patients & 1061 & 203 & 137 & 71 \\
\hline Sex: F/M & $802 / 259$ & $144 / 59$ & $80 / 57$ & $39 / 32$ \\
\hline No. of aneurysms (no. ruptured/unruptured) & $1631(492 / 1139)$ & $249(66 / 183)$ & $147(17 / 130)$ & $102(41 / 61)$ \\
\hline Mean patient age $( \pm$ SD) & $56.25 \pm 13.77$ & $54.68 \pm 13.61$ & $69.11 \pm 10.99$ & $53.07 \pm 11.70$ \\
\hline Patients w/ multiple aneurysms & 329 & 35 & 10 & 16 \\
\hline No. of patients w/ SAH & 490 & 66 & 41 \\
\hline Aneurysm location, no. (\%), no. ruptured/unruptured & & & 28 \\
\hline ACA & $57(3.49), 20 / 37$ & $5(2.00), 2 / 3$ & $2(1.36), 1 / 1$ & $6(5.88), 4 / 2$ \\
\hline ACoA & $226(13.86), 148 / 78$ & $45(18.07), 23 / 22$ & $30(20.41), 3 / 27$ & $21(20.59), 14 / 7$ \\
\hline BA & $106(6.50), 35 / 71$ & $14(5.62), 7 / 7$ & $6(4.08), 1 / 5$ & $7(6.86), 7 / 0$ \\
\hline ICA & $636(38.99), 64 / 572$ & $77(30.92), 5 / 72$ & $15(10.20), 1 / 14$ & $21(20.59), 4 / 17$ \\
\hline MCA & $310(19.01), 82 / 228$ & $71(28.51), 14 / 57$ & $54(36.73), 7 / 47$ & $34(33.33), 13 / 21$ \\
\hline PCoA & $260(15.94), 127 / 133$ & $33(13.25), 14 / 19$ & $40(27.21), 4 / 36$ & $11(10.78), 4 / 7$ \\
\hline VA & $36(2.21), 16 / 20$ & $4(1.61), 1 / 3$ & $0(0), 0 / 0$ & $2(1.96), 2 / 0$ \\
\hline
\end{tabular}

$\mathrm{ACA}=$ anterior cerebral artery; $\mathrm{ACOA}=$ anterior communicating artery; $\mathrm{BA}=$ basilar artery; $\mathrm{ICA}=$ internal carotid artery; $\mathrm{MCA}=$ middle cerebral artery; $\mathrm{PCo} \mathrm{A}=$ posterior communicating artery; $\mathrm{SD}=$ standard deviation; $\mathrm{VA}=$ vertebral artery.

${ }^{*}$ Training population of original model.

the Japanese images, Amira 5.6 (FEI/VSF-division) was used with a thresholding method.

After vessel lumen segmentation, computational meshes of tetrahedral elements with a maximum size of 0.2 $\mathrm{mm}$ were obtained with in-house software for subsequent computational fluid dynamics (CFD) simulations. ${ }^{4}$ Next, the unsteady Navier-Stokes equations were numerically solved with an in-house finite element solver for two cardiac cycles of $60 \mathrm{bpm}$. Inlet and outlet boundary conditions were set as pressure and flow inlets/outlets as previously described. ${ }^{6}$ For all data sets except the Japanese, inlet boundary conditions were imposed at either the internal carotid artery for IAs located at the anterior circulation or the vertebral artery for IAs of the posterior circulation. For the Japanese models, inflow boundary conditions were imposed at the IA's respective parent vessel.

The results of the second cardiac cycle were used to characterize the aneurysm's hemodynamic environment according to 22 hemodynamic parameters capturing flow complexity, concentration, instability, and wall shear stress (WSS) distribution. ${ }^{6}$ The generated computational meshes were also used to characterize the aneurysm shape according to 25 geometric parameters describing aneurysm size, elongation, sphericity, and irregularity. ${ }^{6}$

\section{Model Evaluation and Comparison}

Based on the computed hemodynamic and morphological parameters, as well as aneurysm location in the cerebral vasculature and patient sex and age, the rupture probability model (later referred to as the "US model" because of its training cohort ${ }^{6}$ ) was used to compute the predicted probability of being ruptured for each of the Japanese and Finnish aneurysms. Subsequently, the model's discriminative power was evaluated in terms of the area under the receiver operating characteristic curve (AUC) for the Japanese, Finnish, and combined Finnish and Japanese data sets.
Next, we aimed to assess whether model performance in the Japanese and Finnish population might be improved by including data from these two cohorts for model training. The data sets were split into model training and testing data. For each of the four populations, $10 \%$ of the data was randomly left out of the model training to be later used for testing ("testing data"), and a model including the same variables as the previously developed rupture probability model was trained on the remaining data using logistic group lasso regression. ${ }^{15}$ The procedure of random data splitting and model fitting was repeated 100 times, and the mean and standard deviation of the AUCs when evaluated in the testing data were reported. As the aneurysm rupture risk varies for Finnish and Japanese patients compared to those in other populations, we hypothesized that the effect of certain hemodynamic and geometric parameters or patient characteristics on the rupture risk might vary by population. Therefore, following the same procedure as described above, we also fitted models including interaction terms between hemodynamic, geometric, and patient age and sex variables and the variable "patient population." Such a model interpolates between a global model and a separate model for each population.

\section{Identification of Similar Cases}

Although the logistic regression rupture probability model has a "simple" quantitative outcome when applied to a new case (the predicted probability of being ruptured), interpretation of the model itself and all of its parameters can be difficult. Therefore, as in our external validation of the model using the two European cohorts, ${ }^{7}$ we assessed the performance of the rupture status classification alternatively based on "similar" cases in our database. To apply this approach to the Japanese and Finnish cohorts, similar cases from the combined US and European data sets were defined as IAs with comparable values in maximum oscillatory shear stress (OSImax), nonsphericity index (NSI), 
TABLE 2. Results of model fitting and evaluation for the US model ${ }^{6}$ and retrained models including data from all four populations without and with interaction terms

\begin{tabular}{|c|c|c|c|c|}
\hline Model Type & Training Data & $\begin{array}{l}\text { AUC Training } \\
\text { (mean } \pm \text { SD) }\end{array}$ & Test Data & AUC Test (mean \pm SD) \\
\hline \multirow{4}{*}{ US model } & \multirow{4}{*}{ US $(n=1631)$} & \multirow{4}{*}{0.8553} & EUR $(n=249)$ & 0.8240 \\
\hline & & & JPN $(n=147)$ & 0.6964 \\
\hline & & & FIN ( $n=102)$ & 0.7209 \\
\hline & & & FIN, JPN $(n=249)$ & 0.7389 \\
\hline \multirow{4}{*}{ No interactions } & \multirow{4}{*}{ All $(n=1914)$} & \multirow{4}{*}{$0.8493 \pm 0.0059$} & $10 \%$ US, EUR, FIN, JPN $(n=215)$ & $0.8268 \pm 0.0323$ \\
\hline & & & $10 \%$ JPN $(n=15)$ & $0.6550 \pm 0.3334$ \\
\hline & & & $10 \%$ FIN $(n=11)$ & $0.7550 \pm 0.1646$ \\
\hline & & & $10 \%$ FIN, JPN $(n=26)$ & $0.7494 \pm 0.1371$ \\
\hline \multirow{4}{*}{ Interactions } & \multirow{4}{*}{ All $(n=1914)$} & \multirow{4}{*}{$0.8681 \pm 0.0091$} & $10 \%$ US, EUR, FIN, JPN $(n=215)$ & $0.8308 \pm 0.0309$ \\
\hline & & & $10 \%$ JPN $(n=15)$ & $0.6576 \pm 0.3199$ \\
\hline & & & $10 \%$ FIN $(n=11)$ & $0.8020 \pm 0.1324$ \\
\hline & & & $10 \%$ FIN, JPN $(n=26)$ & $0.8274 \pm 0.1031$ \\
\hline
\end{tabular}

All = data from all four populations without the excluded data for testing; EUR = European; FIN = Finnish; JPN = Japanese; $n=$ number of aneurysms.

aneurysm size, and mean surface curvature (MLN) and located at the same position in the cerebral vasculature. OSImax, NSI, and aneurysm location had previously been identified as important variables for discrimination of aneurysm rupture status. ${ }^{6}$ We added MLN in this study since this parameter was one of the most frequently included parameters in the repeatedly fitted models, whereas interactions between patient population and MLN were included in none of the models. This finding indicates that MLN has an influence on aneurysm rupture status, which does not vary by patient population. The latter aspect was important since we used similar US and European IAs to predict the rupture status of Finnish and Japanese IAs. Aneurysm size was included to capture the IA's current phase of evolution. Details of the definition of rupture status and the exact definition of similar cases can be found in Detmer et al. ${ }^{7}$

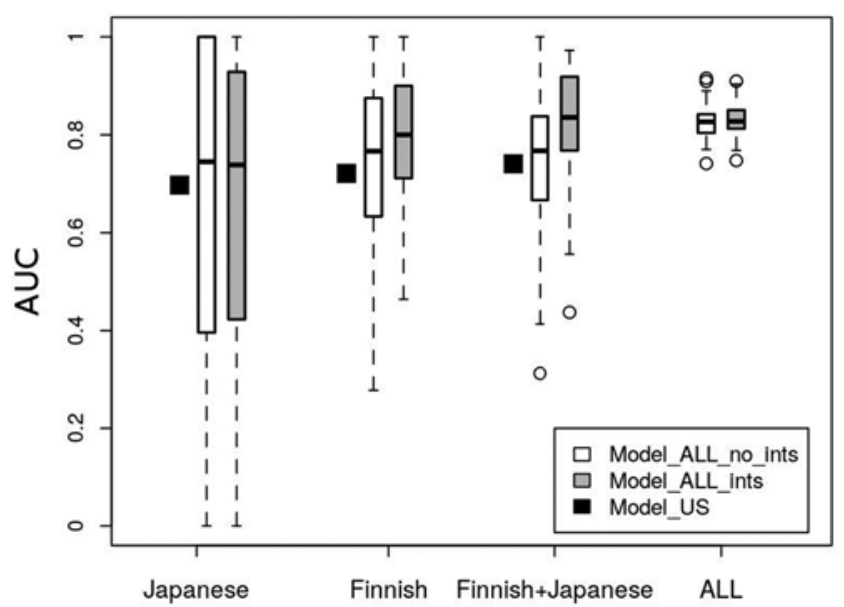

FIG. 1. Box plots showing the AUCs of the repeatedly fitted models when they were evaluated in the Japanese, Finnish, combined Finnish and Japanese, and all testing data. ints = interactions.
All statistical data processing was performed with scripts written in the $\mathrm{R}$ language.

\section{Results}

Table 2 and Fig. 1 show the AUCs when evaluating each of the three categories of models - the previously developed model using the US data (US model) and the model including Japanese and Finnish aneurysms in the training data without and with interaction terms. When evaluating the US model in the Finnish and Japanese data, the AUCs were 0.72 and 0.70 , respectively, indicating considerably lower model performance compared to that in the European cohorts (AUC $=0.82$ ). Figure 2 left compares the ROC curves of the US model for each of the populations, also illustrating its comparatively low performance in the Finnish and Japanese data. The model further seems to be miscalibrated for these two populations as indicated by the calibration plot in the same figure. For a perfectly calibrated fit, all outcomes (grouped into deciles in Fig. 2 right) as well as the loess smoothers (fitted with a span parameter of $0.75^{1}$ ) would lie on the $45^{\circ}$ line. The US model is well calibrated for the training data and for the external European data.

When retraining the model using a subset of data from all four cohorts, the AUC of the model in the Japanese data decreased on average to $0.66( \pm 0.33$; Table 2); for the Finnish IAs, the AUC increased to $0.76( \pm 0.16)$. For the models including interaction terms, the mean AUC remained almost the same for the Japanese cohort $(0.66 \pm$ $0.32)$, but increased greatly for the Finnish data $(0.80 \pm$ $0.13)$ and the combined Finnish and Japanese data $(0.83 \pm$ $0.10)$. In the testing data combined, the AUCs were $0.83 \pm$ 0.03 for the model without interactions and $0.83 \pm 0.03$ for the models including interactions.

Features that were most frequently ( $\geq 99 \%$ ) included for the refitted models without interactions were patient sex and age, aneurysm kinetic energy (KE), low shear area 

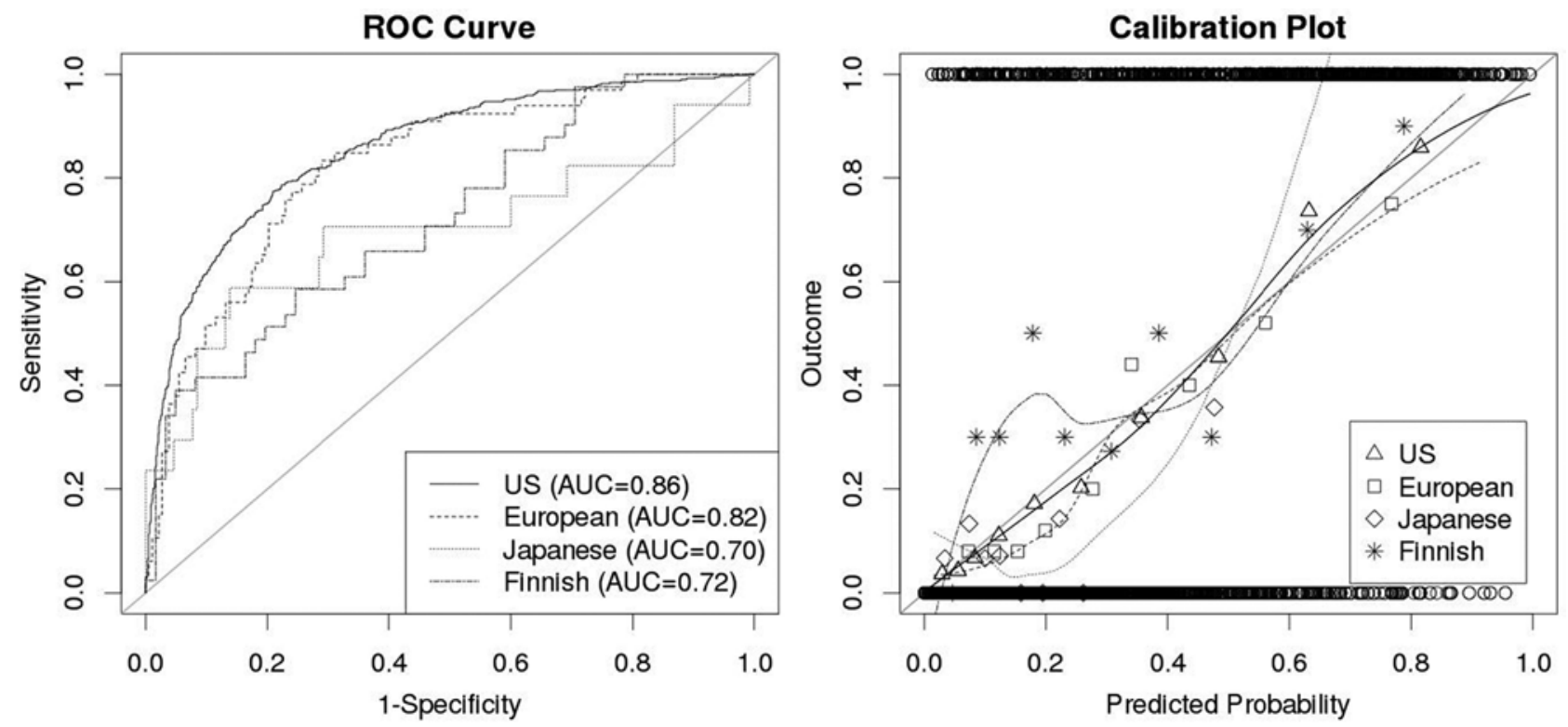

FIG. 2. Receiver operating characteristic (ROC) curves (left) and calibration plots (right) of the US model for the US training data, external European data, and Finnish and Japanese cohorts. ROC curves illustrate the discrimination of a model, while calibration plots allow one to assess a model's goodness of fit. Circles at the top and bottom of the calibration plot show the observed data. The geometric shapes show the observed outcomes of the training and testing populations grouped by deciles, which are also represented by the loess smoother (solid and dotted lines). For a perfectly calibrated fit, all geometric shapes and the loess smoother would lie on the $45^{\circ}$ line.

(LSA), OSImax, height/width ratio (HWR), bulge location (BL), volume/ostium ratio (VOR), NSI, MLN, and aneurysm location. For the models including interaction terms, the most frequently included parameters were patient age, sex, population (note that patient population had been excluded from regularization in the group lasso approach because of the added interaction terms and hence by default was included in all fitted models), KE, LSA, OSImax, and HWR, BL, NSI, MLN, mean Gaussian curvature (GLN), aneurysm location, as well as interactions between patient population and aneurysm neck area (Narea), mean OSI (OSImean), and area weighted average of Gaussian curvature (GAA).

When using similar aneurysms from the European and US populations for classifying Finnish and Japanese aneurysms as ruptured or unruptured, $71 \%$ of the Finnish aneurysms and $81 \%$ of the Japanese aneurysms were correctly classified. The respective true-positive and falsepositive rates were 0.5122 and 0.1639 for the Finnish data and 0.3529 and 0.1308 for the Japanese data.

\section{Discussion}

The treatment decision for incidentally diagnosed IAs poses a challenge for physicians, who must weigh the interventional risk against the risk of potential aneurysmal SAH. A statistical model could support these treatment decisions. An essential requirement for such a model is generalizability to patient cohorts other than the one used for model training. Japanese and Finnish cohorts have a higher aneurysm rupture risk than other populations. ${ }^{11,22}$ The underlying mechanisms of this finding are not yet completely understood. With this study, we aimed to deter- mine whether our model trained on US data and validated on European data would also be applicable to Finnish and Japanese patients. Our results suggest that the relative importance of hemodynamics, aneurysm morphology, and patient-related factors for the eventual risk of rupture differs in aneurysms from the Finnish and Japanese populations, indicating that other environmental or genetic factors cause the increased risk of aneurysmal SAH observed in these two populations. Moreover, our findings demonstrate how rupture probability models for IAs need to be adjusted to specific populations.

\section{Model Performance}

We observed that the model's performance was largely reduced in the Finnish and Japanese data, revealed by the comparatively low AUC (0.74 for combined Finnish and Japanese data) and relatively poor calibration. This finding indicates that different IA characteristics may be associated with rupture in these two populations versus the US and European cohorts. This hypothesis is further supported by our observation that simply including data from Finnish and Japanese patients in the training data did not greatly improve model performance (AUC changed from 0.74 to 0.75 on average), but it increased significantly ( $p<0.0001$, t-test) when the interaction terms of hemodynamic, geometric, and patient-related parameters with patient population were included in the model. Interaction terms that were most frequently included in the fitted models were interactions with aneurysm neck area, mean OSI, area weighted average of Gaussian curvature, and patient sex. Why these particular variables seem to have a different effect on IA rupture status depending on the patient popu- 


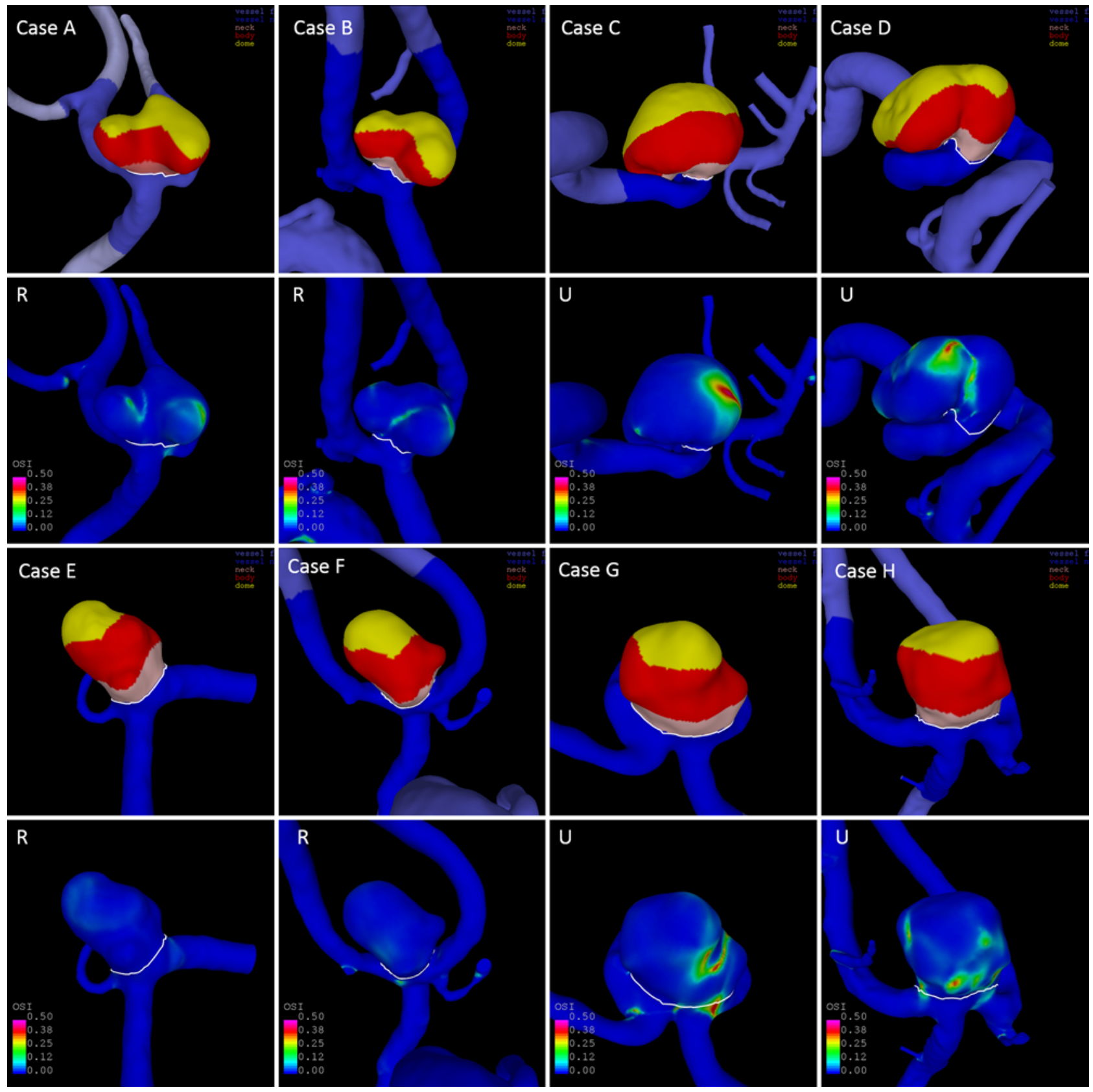

FIG. 3. Computational models showing aneurysm shapes (1st and 3rd rows) and OSI distributions (2nd and 4th rows) of two aneurysms from Finnish patients (cases $A$ and $C$ ), two from Japanese patients (cases $E$ and $G$ ), and respective similar cases from the US-European cohorts (cases B, D, F, and H). The four aneurysms on the left are ruptured (R), and the four aneurysms on the right are unruptured (U). Values for selected parameters in all eight cases are shown in Table 3.

lation needs to be explored further. Possible explanations include different genetic backgrounds that would affect the mechanobiological coupling of hemodynamic factors and vessel wall remodeling, or population-related differences in environmental or other acquired factors that predispose to aneurysm rupture and may explain why aneurysms that would not rupture in other populations would rupture in the Finnish or Japanese.

Other important variables (without interaction terms) included patient age, sex, population, KE, LSA, OSImax, HWR, BL, NSI, MLN, mean Gaussian curvature (GLN), and aneurysm location. In particular, the association of NSI and LSA with IA rupture status is consistent with findings in previous studies. ${ }^{8,9,14,26}$

The poor average performance of all the models with the Japanese data alone (not combined with Finnish) could be explained in part by several technical aspects. First, in contrast to the data from the other patient cohorts, the computational 3D models used for the CFD simulations of the Japanese IAs were cut at the parent vessel of the IA. This might have led to differences in the computed hemodynamics and should be assessed further. This issue is not 

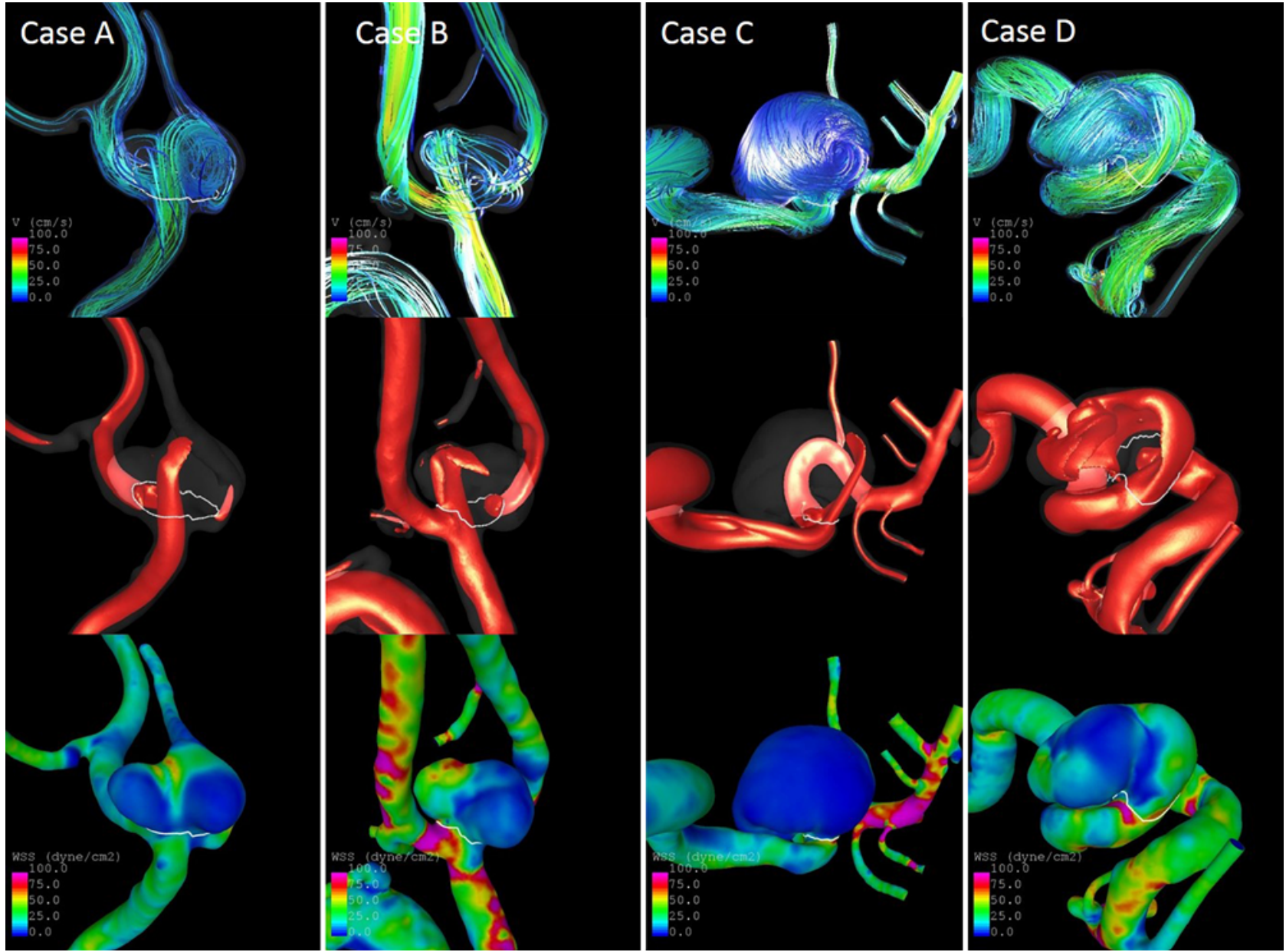

FIG. 4. Streamlines (upper), inflow jets (center), and WSS distributions (lower) at half of the cardiac cycle for the two Finnish aneurysms (cases $A$ and $C$ ) and their similar cases (cases B and D).

expected to greatly affect the similarity-based approach, which performed well in the Japanese data set since it is mainly based on geometric parameters.

Second, unlike the data for the other populations, the Japanese data were longitudinal data obtained by following up patients for a certain time. Consequently, the data set contained a low number of ruptured IAs, particularly in the testing data sets for the repeatedly fitted models. This aspect also explains the large variation in discrimination power for the different testing data sets (standard deviation of 0.32 , range between 0 and 1), meaning that the observed AUC was largely influenced by which particular ruptured IAs were included in the data set for evaluation. When combining the Japanese and Finnish testing data for model evaluation, the mean AUC increased to 0.83 and the standard deviation was reduced to 0.10 . For a robust estimation of the models' performance in Japanese IAs, a larger data set may be needed.

For evaluating the fitted models trained using data from all four populations, a split-sample approach was used since no fully external data were available at this point. Hence, the predictive performance of the fitted models needs to be confirmed with a larger external data set in the future.

Still, the good performance in the Japanese and Finnish testing data combined provides some support to the idea that statistical models based on hemodynamic, morphological, and patient data could improve assessment of future aneurysm rupture risk since the Japanese data set was longitudinal (based on follow-up data).

\section{Aneurysm Rupture Status Classification Based on Similar Cases}

The identification of similar IAs to classify a new IA's rupture status had good results with accuracies of $71 \%$ in the Finnish cohort and $81 \%$ in the Japanese cohort. The models and OSI distributions of eight examples are illustrated in Fig. 3. Additional flow characteristics (WSS, inflow jets, and streamlines) of the eight aneurysms are shown in Figs. 4 and 5. Aneurysms A and C are from Finnish patients, and similar cases B and D were identified from the US and European data sets using the algorithm described above. For the two aneurysms in Japanese patients (cases $\mathrm{E}$ and $\mathrm{G}$ ), the similar cases $\mathrm{F}$ and $\mathrm{H}$ were matched. All "similar" aneurysms have a comparable shape and OSI distribution. Values for selected parameters in these eight aneurysms are shown in Table 3. The Finnish and Japanese and their respective US-European cases had, by definition, close values in NSI, OSImax, MLN, and aneurysm size. 

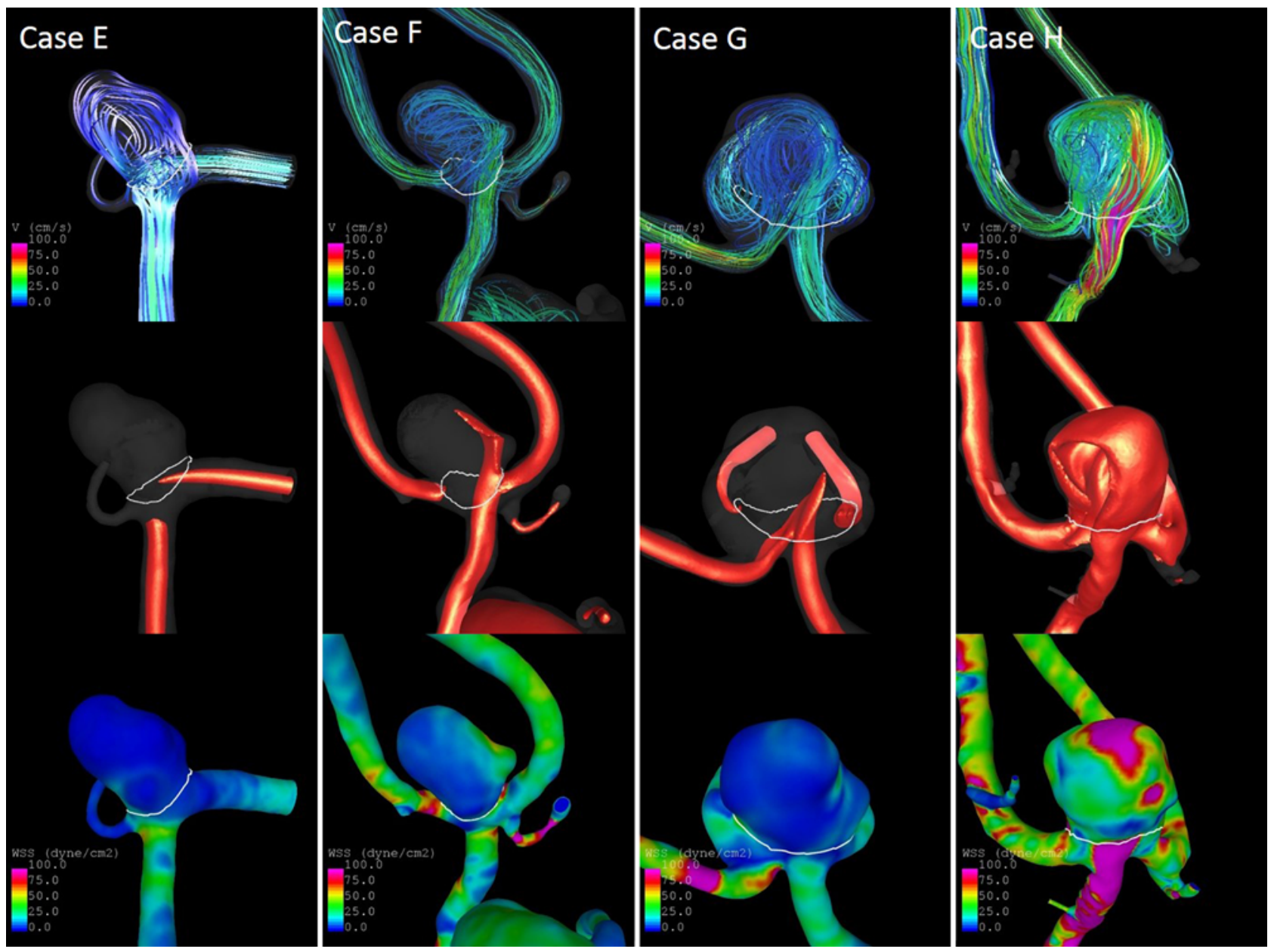

FIG. 5. Streamlines (upper), inflow jets (center), and WSS distributions (lower) at half of the cardiac cycle for the two Japanese aneurysms (cases $E$ and $G$ ) and their similar cases (cases $F$ and $H$ ).

Furthermore, they had overall similar predicted probabilities of being ruptured based on the US model. Interestingly, aneurysm $\mathrm{G}$-consistent with its rupture status - had a lower probability (26\%) of being ruptured than the four ruptured cases despite being relatively large and located at the anterior communicating artery, which are characteris- tics typically associated with high-risk aneurysms. Thus, aneurysm $G$ represents a case in which an aneurysm that may have been intuitively thought of as high risk could actually be a low-risk aneurysm (this aneurysm remained unruptured during the longitudinal follow-up), which is correctly detected by the model. Consistently, it was also

TABLE 3. Values of selected variables and predicted probabilities of being ruptured based on the statistical model for the IAs illustrated in Fig. 3

\begin{tabular}{ccclccccc}
\hline Case & Population & Asize $(\mathrm{cm})$ & Location & NSI & OSImax & MLN & Predicted Probability* & Rupture Status $†$ \\
\hline A & FIN & 0.6922 & ACoA & 0.2443 & 0.2703 & 0.3663 & 0.6609 & R \\
\hline B & US & 0.6797 & ACoA & 0.2405 & 0.2892 & 0.3821 & 0.7329 & R \\
\hline C & FIN & 1.6644 & ICA-CAV & 0.2498 & 0.4665 & 0.4504 & 0.0713 & U \\
\hline D & EUR & 1.5896 & ICA-CAV & 0.2420 & 0.4845 & 0.4661 & 0.0327 & U \\
\hline E & JPN & 0.7339 & ACoA & 0.2168 & 0.3114 & 0.3560 & 0.5550 & R \\
\hline F & US & 0.7520 & ACoA & 0.2218 & 0.3196 & 0.3628 & 0.6142 & R \\
\hline G & JPN & 0.8989 & ACoA & 0.1737 & 0.3973 & 0.3256 & 0.2636 & 0.5837 \\
\hline H & US & 0.8146 & ACoA & 0.1683 & 0.4033 & 0.3435 & U \\
\hline
\end{tabular}

Asize = aneurysm size; $I C A-C A V=$ cavernous sinus of the internal carotid artery; $R=$ ruptured; $U=$ unruptured.

* Predicted probability of being ruptured based on the rupture probability model. ${ }^{6}$

$\dagger$ Rupture status refers to the true rupture status of the aneurysm. 
matched with an unruptured IA. The similarity-based approach could potentially be improved by taking into account other IA characteristics. For example, the configuration of an IA's parent arteries is not considered, although it does influence the inflow into the aneurysm. Additionally, similarity in terms of flow was only captured by OSImax, resulting in, for example, different WSS distributions for some cases (see WSS distribution for cases $\mathrm{G}$ and $\mathrm{H}$ in Fig. 5). The good performance with the Japanese data may also have resulted in part from the low number of ruptured aneurysms and needs to be further explored with data from more aneurysms.

Nonetheless, the definition of rupture status based on similar cases in the database performed well overall, especially with the Japanese data. In a clinical setting, it could be used as an illustrational tool, perhaps in addition to a statistical model.

\section{Clinical Considerations}

For eventual translation of this statistical model for aneurysm rupture into the clinic, several additional steps are needed. First, since the models were mainly developed based on cross-sectional data, they provide the probability of whether a given IA is currently ruptured or unruptured. To assess whether the models could also be used for the assessment of a risk of future rupture-i.e., whether the underlying assumption that high-risk cases resemble those that have already ruptured holds $s^{5,6,25}$ - they need to be evaluated with more longitudinal data. Second, this study demonstrates how population affects the rupture probability models of IAs, and thus such models must be tested in specific populations separately. Third, to be able to apply such a model in a clinical setting, clinicians would need to be provided with a tool for image-based IA shape characterization as well as CFD simulations, which would seem to be feasible in the future. ${ }^{24}$ Finally, the model could potentially be improved by taking into account additional patient-related information such as comorbidities or social habits including smoking or alcohol consumption.

\section{Conclusions}

In order to develop an aneurysm rupture prediction model based on aneurysm geometry, hemodynamics, and patient characteristics that applies to Japanese and Finnish aneurysms, the inclusion of data from these two cohorts for model training, as well as interaction terms between patient population and the other variables in the statistical model, is necessary. When including this information, the performance of such a model with Japanese and Finnish data is close to its performance with US or European data. These results suggest that population-specific differences determine how hemodynamics and shape associate with rupture risk in IAs.

\section{Acknowledgments}

The European (other than Finnish) data included the publicly available AneuRisk data set and the AneuX data set, collected and processed in the context of the @neurIST project funded by the European Commission (IST-2004-027703), and the AneuX project was evaluated by the Swiss National Science Foundation and funded by the SystemsX.ch initiative (MRD 2014/261). This study received funding from the Finnish Medical Foundation (J.F.) and research grants from the Kuopio University Hospital. This work was also supported by NIH grant no. R01NS097457 (J.R.C.).

\section{References}

1. Austin PC, Steyerberg EW: Graphical assessment of internal and external calibration of logistic regression models by using loess smoothers. Stat Med 33:517-535, 2014

2. Backes D, Rinkel GJE, Greving JP, Velthuis BK, Murayama Y, Takao H, et al: ELAPSS score for prediction of risk of growth of unruptured intracranial aneurysms. Neurology 88:1600-1606, 2017

3. Cebral JR, Castro MA, Appanaboyina S, Putman CM, Millan D, Frangi AF: Efficient pipeline for image-based patient-specific analysis of cerebral aneurysm hemodynamics: technique and sensitivity. IEEE Trans Med Imaging 24:457-467, 2005

4. Cebral JR, Raschi M: Suggested connections between risk factors of intracranial aneurysms: a review. Ann Biomed Eng 41:1366-1383, 2013

5. Chung BJ, Mut F, Putman CM, Hamzei-Sichani F, Brinjikji W, Kallmes D, et al: Identification of hostile hemodynamics and geometries of cerebral aneurysms: a case-control study. AJNR Am J Neuroradiol 39:1860-1866, 2018

6. Detmer FJ, Chung BJ, Mut F, Slawski M, Hamzei-Sichani F, Putman C, et al: Development and internal validation of an aneurysm rupture probability model based on patient characteristics and aneurysm location, morphology, and hemodynamics. Int J CARS 13:1767-1779, 2018

7. Detmer FJ, Fajardo-Jiménez D, Mut F, Juchler N, Hirsch S, Pereira VM, et al: External validation of cerebral aneurysm rupture probability model with data from two patient cohorts. Acta Neurochir (Wien) 160:2425-2434, 2018

8. Dhar S, Tremmel M, Mocco J, Kim M, Yamamoto J, Siddiqui $\mathrm{AH}$, et al: Morphology parameters for intracranial aneurysm rupture risk assessment. Neurosurgery 63:185-197, 2008

9. Duan G, Lv N, Yin J, Xu J, Hong B, Xu Y, et al: Morphological and hemodynamic analysis of posterior communicating artery aneurysms prone to rupture: a matched case-control study. J Neurointerv Surg 8:47-51, 2016

10. Etminan N, Brown RD Jr, Beseoglu K, Juvela S, Raymond $\mathrm{J}$, Morita A, et al: The unruptured intracranial aneurysm treatment score: a multidisciplinary consensus. Neurology 85:881-889, 2015

11. Greving JP, Wermer MJ, Brown RD Jr, Morita A, Juvela S, Yonekura M, et al: Development of the PHASES score for prediction of risk of rupture of intracranial aneurysms: a pooled analysis of six prospective cohort studies. Lancet Neurol 13:59-66, 2014

12. Juvela S, Poussa K, Lehto H, Porras M: Natural history of unruptured intracranial aneurysms: a long-term follow-up study. Stroke 44:2414-2421, 2013

13. Kleinloog R, de Mul N, Verweij BH, Post JA, Rinkel GJE, Ruigrok YM: risk factors for intracranial aneurysm rupture: a systematic review. Neurosurgery 82:431-440, 2018

14. Lv N, Wang C, Karmonik C, Fang Y, Xu J, Yu Y, et al: Morphological and hemodynamic discriminators for rupture status in posterior communicating artery aneurysms. PLoS One 11:e0149906, 2016

15. Meier L, Van De Geer S, Bühlmann P: The group lasso for logistic regression. J R Stat Soc Series B Stat Methodol 70:53-71, 2008

16. Meng H, Tutino VM, Xiang J, Siddiqui A: High WSS or low WSS? Complex interactions of hemodynamics with intracranial aneurysm initiation, growth, and rupture: toward a unifying hypothesis. AJNR Am J Neuroradiol 35:1254-1262, 2014 
17. Morita A, Kirino T, Hashi K, Aoki N, Fukuhara S, Hashimoto N, et al: The natural course of unruptured cerebral aneurysms in a Japanese cohort. N Engl J Med 366:2474-2482, 2012

18. Rinkel GJ, Djibuti M, Algra A, van Gijn J: Prevalence and risk of rupture of intracranial aneurysms: a systematic review. Stroke 29:251-256, 1998

19. Rivero-Arias O, Gray A, Wolstenholme J: Burden of disease and costs of aneurysmal subarachnoid haemorrhage (aSAH) in the United Kingdom. Cost Eff Resour Alloc 8:6, 2010

20. Vlak MH, Algra A, Brandenburg R, Rinkel GJ: Prevalence of unruptured intracranial aneurysms, with emphasis on sex, age, comorbidity, country, and time period: a systematic review and meta-analysis. Lancet Neurol 10:626-636, 2011

21. Wang G, Zhang Z, Ayala C, Dunet DO, Fang J, George MG: Costs of hospitalization for stroke patients aged 18-64 years in the United States. J Stroke Cerebrovasc Dis 23:861-868, 2014

22. Wermer MJ, van der Schaaf IC, Algra A, Rinkel GJ: Risk of rupture of unruptured intracranial aneurysms in relation to patient and aneurysm characteristics: an updated metaanalysis. Stroke 38:1404-1410, 2007

23. Wiebers DO, Whisnant JP, Huston J III, Meissner I, Brown RD Jr, Piepgras DG, et al: Unruptured intracranial aneurysms: natural history, clinical outcome, and risks of surgical and endovascular treatment. Lancet 362:103-110, 2003

24. Xiang J, Varble N, Davies JM, Rai AT, Kono K, Sugiyama SI, et al: Initial clinical experience with AView-a clinical computational platform for intracranial aneurysm morphology, hemodynamics, and treatment management. World Neurosurg 108:534-542, 2017

25. Xiang J, Yu J, Choi H, Dolan Fox JM, Snyder KV, Levy EI, et al: Rupture Resemblance Score (RRS): toward risk stratification of unruptured intracranial aneurysms using hemodynamic-morphological discriminants. J Neurointerv Surg 7:490-495, 2015

26. Zhang Y, Yang X, Wang Y, Liu J, Li C, Jing L, et al: Influence of morphology and hemodynamic factors on rupture of multiple intracranial aneurysms: matched-pairs of rupturedunruptured aneurysms located unilaterally on the anterior circulation. BMC Neurol 14:253, 2014

\section{Disclosures}

The authors report no conflict of interest concerning the materials or methods used in this study or the findings specified in this paper.

\section{Author Contributions}

Conception and design: Detmer, Mut, Slawski, Frösen, Cebral. Acquisition of data: Detmer, Hadad, Chung, Mut, Juchler, Bijlenga, Uchiyama, Fujimura, Murayama, Koivisto, Frösen, Cebral. Analysis and interpretation of data: Detmer, Hadad, Mut, Slawski, Juchler, Hirsch, Bijlenga, Frösen, Cebral. Drafting the article: Detmer, Hadad. Critically revising the article: all authors. Reviewed submitted version of manuscript: Detmer, Hadad, Chung, Mut, Slawski, Juchler, Kurtcuoglu, Hirsch, Bijlenga, Uchiyama, Fujimura, Yamamoto, Murayama, Takao, Koivisto, Cebral. Approved the final version of the manuscript on behalf of all authors: Detmer. Statistical analysis: Detmer, Hadad, Slawski. Study supervision: Chung, Slawski, Bijlenga, Yamamoto, Takao, Frösen, Cebral. Supervising student: Kurtcuoglu.

\section{Supplemental Information}

\section{Previous Presentations}

Partial preliminary results of this work have been submitted as an abstract that will be presented at the Summer Biomechanics, Bioengineering, and Biotransport Conference held in Seven Springs, Pennsylvania, on June 25-28, 2019.

\section{Correspondence}

Felicitas J. Detmer: George Mason University, Fairfax, VA. fdetmer@gmu.edu. 